\title{
Predictive and prognostic value of classification by immuno- histochemical phenotypes in localised breast cancer treated with dose-dense adriamycin/cyclophosphamide
}

\author{
Zarcos Pedrinaci Irene ${ }^{1,2, *}$, Redondo Maximino ${ }^{2,3}$, Calvo Ángel ${ }^{4}$, Rivas-Ruiz Francisco ${ }^{2,3}$, Puche JLuis ${ }^{5}$ and Rueda Antonio ${ }^{1,2}$ \\ 1 Unit of Medical Oncology, Hospital Costa del Sol, Autovía A-7. Km 187, 29600 Marbella, Málaga, Spain \\ 2 Red de investigación en servicios de salud en enfermedades crónicas REDISSEC, Barcelona, Spain \\ ${ }^{3}$ Research Unit, Hospital Costa del Sol, University of Málaga, Autovía A-7, KM187, 29600 Marbella, Málaga, Spain \\ ${ }^{4}$ Radiation Oncology Unit, Hospital Clínico San Cecilio de Granada, Granada, Spain \\ ${ }^{5}$ Department of Medical Oncology, Hospital. Clinical Hospital San Cecilio de Granada, Granada, Spain
}

\begin{abstract}
Introduction: Histopathologic subtyping according to the St. Gallen classification is predictive of response in women with breast cancer treated with neoadjuvant chemotherapy (NCT). Our aim was to confirm its predictive role when dose-dense (DD) chemotherapy is administered. Methods: A prospective study was performed in stage II-III breast cancer treated with four cycles of doxorubicin-cyclophosphamide every two weeks, followed by 4 cycles of docetaxel every 3weeks. Tumours were grouped into 4 subtypes. Simple and multivariate logistic regression analysis was performed to determine the association between pathologic complete response (pCR), according to the Miller \& Payne classification and tumour subtype. In addition survival analysis was performed. Results: 71 patients were included. By phenotypic classification, $11.3 \%$ were Luminal A, 59.2\% Luminal B, 8.5\% pure HER2 and $21.1 \%$ TN. Following the administration of DD NCT, a pCR rate of $25.4 \%$ was achieved. There was a significant association between the $\mathrm{pCR}$ rate and the different immune phenotypes ( $p=0.05)$. The pCR rate was $46.7 \%$ for the TN patients and $19.6 \%$ for the others $(p=0.046)$. At 30 months' follow up, the rate of disease-free survival (DFS) was $71.8 \%$ for the TN patients and $93.3 \%$ for all others. The difference was also significant for overall survival $(p=0.006)$, with $100 \%$ of the non-TN patients alive vs $85.7 \%$ of the TN patients. Within the TN subtype, the patients who achieved $p C R$ had a better DFS ( $p=0.04$ ) and a better rate of overall survival. Conclusions: Tumour classification according to the St. Gallen phenotypes is useful in predicting response and survival in breast cancer patients treated with DD neoadjuvant chemotherapy.
\end{abstract}

Keywords: dose-dense; neoadjuvant; breast cancer; pathologic-response; phenotype; prognostic

\section{Introduction}

Since the early 1980 s there has been increasing interest in the application of neoadjuvant chemotherapy (NCT) in cases of unresectable or locally advanced breast cancer. This interest was based on the hypotheses proposed following laboratory findings [1,2] and in view of the favourable results obtained from a series of clinical trials and a meta-analysis which showed that this treatment sequence, for overall survival (OS) and disease-free survival (DFS), obtained the same results as chemotherapy after surgery [3-5].

NCT has several advantages over post-operative application at an early stage: it acts against micrometastatic disease and at the same time reduces the tumour size, thus permitting more conservative surgery to be undertaken. In addition, the sensitivity of the tumour to systemic therapy can be tested $[3,6,7]$. Furthermore, the pathologic tumour response to preoperative chemotherapy can provide a useful forecasting tool as it has been shown that pathologic complete response ( $p C R$ ) is associated with a lower risk of relapse and with increased OS [8]. Therefore, NCT in breast cancer is now widely employed at early stages of the disease, and obtaining a high rate of $\mathrm{pCR}$ has become the main goal in most clinical trials of neoadjuvant therapy.

The classification of breast cancer into molecular subtypes by Perou et al. has a proven forecast value and plays an important role as a predictor of response to NCT. Later studies using a 3-5 marker panel determined by immunohistochemical techniques (see Method section),

*Corresponding author: Zarcos Pedrinaci Irene, Unit of Medical Oncology, Hospital Costa del Sol, Autovía A-7. Km 187, 29600 Marbella, Málaga, Spain and Researcher at the Research Network on Health Services and Chronic Diseases- REDISSEC. Tel.: 0034 951976620; Email: irenezarcos@hotmail. com

Received 21 August 2015 Revised 2 November 2015 Accepted 10 November 2015 Published 18 November 2015

Citation: Pedrinaci IZ, Maximino R, Ángel C, FranciscoRR, Luis JP, Antonio R. Predictive and prognostic value of classification by immunohistochemical phenotypes in localised breast cancer treated with dose-dense adriamycin/cyclophosphamide. J Cancer Res Ther. 2015; 3(11):129-135. DOI:10.14312/2052-4994.2015-19

Copyright: (C) 2015 Pedrinaci IZ, et al. Published by NobleResearch Publishers. This is an open-access article distributed under the terms of the Creative Commons Attribution License, which permits unrestricted use, distribution and reproduction in any medium, provided the original author and source are credited. 
have also sought to classify breast cancer and provide a similar interpretation [9].

With the aim of optimising the application of NCT in cases of locally advanced breast cancer, our study analysed the effectiveness of dose-dense (DD) NCT. So the primary aim was to determine the relationship between the different breast cancer subtypes, according to the St. Gallen classification, and the response to DD NCT, according to the rate of $\mathrm{pCR}$ on the Miller \& Payne classification. As a secondary goal, we analysed the prognostic value of this sub-classification of breast cancer immunophenotypes treated with DD NCT, evaluating DFS and OS.

\section{Key messages}

Tumour classification according to the St. Gallen phenotypes is useful in predicting response and survival in breast cancer patients treated with DD neoadjuvant chemotherapy. TN tumors had best pRC rates and less DFS and OS than other breast cancer subtypes. pRC in TN tumours had a prognostic value.

\section{Subjects and methods}

\section{Assessment criteria}

Breast cancer subtypes were determined in accordance with the clinical-pathologic criteria recommended in the 2011 St. Gallen consensus [10]. This was done using a combination of markers, including the Ki-67 proliferation index and immunohistochemical markers based on the expression pattern of estrogen receptors (ER), progesterone receptors (PR) and HER2. If the HER2 status was equivocal $(2+)$ or the samples suboptimal, this pattern was determined by fluorescence in situ hybridisation (FISH) $[11,12]$. Four major subtypes were identified: luminal $A$ tumours, with ER and PR positive, HER2 negative and Ki$67<14 \%$; luminal B, with ER and PR positive and Ki-67>14\% and/or HER2 amplified; pure HER2, with overexpressed HER2, and ER and PR negative; and triple negative (TN), with an absence of expression for all three receptors.

The pathologic tumour response to NCT was evaluated according to the Miller \& Payne scale, graduated from 1 to 5 [13]. The response was classified as pCR grade 5 vs. non$\mathrm{pCR}$, which includes grades 1 to 4 .

The relationship between the different phenotypes of breast cancer and OS and DFS was analysed according to the pathologic response achieved. DFS was defined as the time elapsed in months between surgery and locoregional or distant relapse. OS was calculated as the difference between the date of diagnosis and the date of death from any cause.

\section{Patients and treatment}

This prospective, observational study of 71 patients diagnosed with stage II-III breast cancer was conducted from May 2011 until February 2015.

The following inclusion criteria were applied: patient aged at least 18 years, diagnosed with stage II or III breast cancer, histologically confirmed by a biopsy of the breast. All cases were discussed in a joint multidisciplinary session before inclusion in the dose-dense NCT programme. A tumour was considered HER2 positive when it presented overexpression of $3+$ by immunohistochemistry or a positive FISH result (technique routinely applied when HER2 status was equivocal $(2+)$ by immunohistochemistry analysis). Exclusion criteria were the presence of metastasis, a previous history of cancer (excluding cervix carcinoma in situ and non-melanoma skin cancer), poor bone marrow function (defined as an absolute neutrophil count $<1,500 / \mu \mathrm{L}$ or platelets $<100,000 / \mu \mathrm{L}$ ), abnormal renal function (defined as serum creatinine $>2.5 \mathrm{mg} / 100 \mathrm{ml}$ ), abnormal hepatic function or a history of congestive heart failure or left ventricular ejection fraction $<45 \%$ (evaluated by echocardiography).

The DD NCT consisted of four cycles of doxorubicin (A) $60 \mathrm{mg} / \mathrm{m}^{2}$ and cyclophosphamide (C) $600 \mathrm{mg} / \mathrm{m}^{2}$ every 2 weeks, with the support of filgrastim $5 \mu / \mathrm{kg}$, from days 3 to 10 of each cycle, followed by 4 cycles of docetaxel (T) 100 $\mathrm{mg} / \mathrm{m}^{2}$ every 3 weeks. Trastuzumab $6 \mathrm{mg} / \mathrm{kg}$ was added (with a loading dose of $8 \mathrm{mg} / \mathrm{kg}$ ) every 3 weeks during the 4 cycles of docetaxel, if HER2 was overexpressed, and was continued after surgery for one year. All tumours with positive ER or PR received adjuvant tamoxifen or aromatase inhibitors after surgery, for 5 years.

After the 8 cycles of NCT, a mammography or breast MRI was performed to evaluate the clinical-radiological response to treatment. The type of surgery (conservative or mastectomy) was scheduled according to the surgeon's criteria.

When clinical examination and ultrasound diagnosis of the axilla were negative, the patient underwent selective sentinel node biopsy before starting NCT. If the result of these tests was positive, an axillary lymphadenectomy was performed during the mammary intervention. All patients given breast-conserving surgery received radiation therapy with whole-breast irradiation, of 40.05 Gy in 15 fractions with breast boost irradiation of $10 \mathrm{~Gy}$ for patients aged under 50 years, or with microscopically affected margins. Irradiation of the breast and/or chest wall and/ or supraclavicular and axillary area was performed, at the same dose, if a residual tumour $>5 \mathrm{~cm}$ and/or pathologic tumour involvement of more than 3 axillary nodes was observed after chemotherapy.

The patients' medical records were reviewed and all clinical, histopathologic and treatment-related variables were included in a database.

\section{Statistical analysis}

SPSS 14.0 (SPSS) statistical software was used to perform a descriptive analysis of the main variables. Measures of central tendency, dispersion and position were calculated for the numeric variables, and absolute and relative frequencies were obtained for the qualitative ones. Student's t test for independent samples was applied in the case of numeric variables, and the chi-square test for the qualitative ones. In addition, the hypothesis of normal distribution of the data was verified, so that non-parametric techniques could be used if necessary. Bivariate analysis was used to study the relationship between, on the one 
hand, the variables known to be predictive and prognostic and related to the pathologic response to treatment, and on the other, the disease-free interval and overall survival achieved stratified multivariate survival analysis was performed, using the Kaplan-Meier method, for DFS and OS using the log-rank test to compare the groups. Cox's regression model was used to perform a stratified analysis of $\mathrm{pCR}$. In all analyses, the level of significance was set at $p<0.05$, with a confidence interval of $95 \%$ where necessary.

\section{Research ethics}

The study was approved by the research ethics committee at the San Cecilio Clinical Hospital (Granada) and was conducted in accordance with the Helsinki Declaration principles and recommendations for biomedical research involving human subjects (General Assembly of the World Medical Association, Helsinki, Finland, 1964). All patients gave their signed informed consent to participate in the study.

\section{Results}

The original study population included 75 women with localised-locally advanced breast cancer, of whom 71 formed the final sample. Four patients were excluded from analysis. In 3 cases, this was because metastatic disease was detected in the extension study before starting NCT, no surgery was performed, and therefore the PCR could not be analysed. In the other case, the patient's chemotherapy regimen was changed. The average age of the population was 49 years. $75 \%$ of the tumours detected were stage T2 or T3, and $57 \%$ presented clinical or cytological lymph node involvement ( $50 \%$ cN1). The phenotypic classification by IHC showed that $70 \%$ of the tumours were luminal $(59 \%$ type B), 9\% were pure HER2 and $21 \%$ were TN. $97 \%$ of the population completed the NCT regimen. Moreover, due to toxicity, 32\% did not complete the four cycles of AC every two weeks, and so the intercycle interval was extended to 21 days in the final cycles of AC. The main clinical, pathologic and treatment-related characteristics of the patients are shown in (Table 1).

Relation between histopathologic subtypes and response to dose-dense neoadjuvant chemotherapy

A tumor pCR rate of $25.4 \%$ (18/71) was recorded. According to the bivariate analysis, the variables that were significantly related to the pCR were the 2011 St. Gallen surrogate classification $(p=0.05)$, and the phenotypes that produced the greatest response were pure HER2 (50\% pCR) and TN (46.7\% pCR); HER2 overexpression $(p=0.016)$; ER negative $(p=0.013)$; PR negative $(p=0.001)$. No multivariate analysis was performed, because the clinical variables related to PCR were some of those used to group the subtypes of breast cancer.

When histopathologic subtypes were grouped and compared the responses of TN vs the rest of tumors, the TN tumours were significantly different, with a higher rate of pCR (46.7\%) than the remaining luminal tumours and HER2 (cPR rate: $19.6 \%)(p=0.046)$. The pure HER2, when analysed separately, did not present statistically significant differences, due to the small number of patients in this subgroup (Table 2).
Table 1 Clinical-pathologic and treatment characteristics. *ki-67\%: In 5 cases, the outcome could not be determined in the pre-NCT biopsy.

\begin{tabular}{|c|c|c|c|}
\hline & & $\begin{array}{l}\text { No. of } \\
\text { patients } \\
(n=71)\end{array}$ & (\%) \\
\hline \multirow[t]{2}{*}{ Menopausal status } & Pre & 40 & 56.3 \\
\hline & Post & 31 & 43.7 \\
\hline \multirow[t]{4}{*}{ TNM classification T } & 1 & 9 & 12.7 \\
\hline & 2 & 37 & 52.1 \\
\hline & 3 & 16 & 22.5 \\
\hline & 4 & 9 & 12.7 \\
\hline \multirow[t]{4}{*}{ TNM classification N } & 0 & 32 & 45 \\
\hline & 1 & 34 & 47.8 \\
\hline & 2 & 4 & 5.6 \\
\hline & 3 & 1 & 1.4 \\
\hline \multirow[t]{3}{*}{ Histologic type } & Ductal & 65 & 91.5 \\
\hline & Lobular & 5 & 7.0 \\
\hline & Mucinous & 1 & 1.4 \\
\hline \multirow[t]{4}{*}{ Phenotype classification } & Luminal A & 8 & 11.3 \\
\hline & Luminal B & 42 & 59.2 \\
\hline & HER2 & 6 & 8.5 \\
\hline & Triple negative & 15 & 21.1 \\
\hline \multirow[t]{3}{*}{ Grade of differentiation } & Good & 15 & 25.4 \\
\hline & Intermediate & 34 & 57.6 \\
\hline & High & 10 & 16.9 \\
\hline \multirow[t]{2}{*}{ Ki-67\%* } & $<14 \%$ & 9 & 12.7 \\
\hline & $>14 \%$ & 57 & 80.3 \\
\hline \multirow[t]{2}{*}{ Estrogen receptors } & Negative & 21 & 29.6 \\
\hline & Positive & 50 & 70.4 \\
\hline \multirow[t]{2}{*}{ Progesterone receptors } & Negative & 30 & 42.3 \\
\hline & Positive & 41 & 57.7 \\
\hline \multirow[t]{2}{*}{ HER2 } & Negative & 56 & 78.9 \\
\hline & Positive & 15 & 21.1 \\
\hline \multirow[t]{2}{*}{ Completed chemotherapy } & No & 2 & 2.8 \\
\hline & Yes & 69 & 97.2 \\
\hline \multirow[t]{2}{*}{ Neoadjuvant trastuzumab } & No & 57 & 80.3 \\
\hline & Yes & 13 & 18.3 \\
\hline \multirow[t]{2}{*}{ Type of surgery } & Tumourectomy & 52 & 73.2 \\
\hline & Mastectomy & 19 & 26.8 \\
\hline \multirow{3}{*}{$\begin{array}{l}\text { Selective sentinel } \\
\text { node biopsy before } \\
\text { chemotherapy }\end{array}$} & Negative & 20 & 28.2 \\
\hline & Positive & 35 & 49.3 \\
\hline & Not stated & 16 & 22.5 \\
\hline \multirow[t]{2}{*}{ Lymphadenectomy } & Yes & 58 & 81.7 \\
\hline & No & 13 & 18.3 \\
\hline
\end{tabular}


Table 2 Relationship between subtypes of breast cancer and immunohistochemical variables to CPR to dose-dense NCT.

\begin{tabular}{|c|c|c|c|c|}
\hline & \multirow{2}{*}{$\mathrm{n}=71(100 \%)$} & \multicolumn{2}{|c|}{$\begin{array}{c}\text { Miller \& Payne } \\
\text { pathologic } \\
\text { response } n(\%)^{*}\end{array}$} & \multirow{2}{*}{$P$ value } \\
\hline & & $\begin{array}{l}\text { Grade } 5 \\
\text { n (\%) } 18 \\
(25.4 \%)\end{array}$ & $\begin{array}{c}\text { Grades } \\
1-4 \mathrm{n}(\%) \\
53(74.6 \%)\end{array}$ & \\
\hline \multirow[t]{8}{*}{ Phenotypes } & Luminal A & 1 & 7 & 0.05 \\
\hline & 8 & $12.5 \%$ & $87.5 \%$ & \\
\hline & Luminal B & 7 & 35 & \\
\hline & 42 & $16.7 \%$ & $83.3 \%$ & \\
\hline & HER2 & 3 & 3 & \\
\hline & 6 & $50 \%$ & $50 \%$ & \\
\hline & Triple negative & 7 & 8 & \\
\hline & 15 & $46.7 \%$ & $53.3 \%$ & \\
\hline \multirow[t]{4}{*}{ Phenotypes } & Luminal+HER2 & 11 & 4 & 0.046 \\
\hline & 56 & $19.6 \%$ & $80.4 \%$ & \\
\hline & Triple negative & 7 & 8 & \\
\hline & 15 & $46.7 \%$ & $53.3 \%$ & \\
\hline \multirow[t]{4}{*}{ HER2 } & Negative & 10 & 46 & 0.016 \\
\hline & & $17.9 \%$ & $82.1 \%$ & \\
\hline & Positive & 8 & 7 & \\
\hline & & $53.3 \%$ & $46.7 \%$ & \\
\hline \multirow{4}{*}{$\begin{array}{l}\text { Estrogen } \\
\text { receptors }\end{array}$} & Negative & 10 & 11 & 0.013 \\
\hline & & $47.6 \%$ & $52.4 \%$ & \\
\hline & Positive & 8 & 42 & \\
\hline & & $16 \%$ & $84 \%$ & \\
\hline \multirow{4}{*}{$\begin{array}{l}\text { Progesterone } \\
\text { receptors }\end{array}$} & Negative & 14 & 16 & 0.001 \\
\hline & & $46.7 \%$ & $53.3 \%$ & \\
\hline & Positive & 4 & 37 & \\
\hline & & $9.8 \%$ & $90.2 \%$ & \\
\hline \multirow[t]{4}{*}{$\mathrm{Ki}-67$} & $<14 \%$ & 3 & 6 & 0.684 \\
\hline & & $33.3 \%$ & $66.7 \%$ & \\
\hline & $>14 \%$ & 14 & 43 & \\
\hline & & $24.6 \%$ & $75.4 \%$ & \\
\hline
\end{tabular}

No significant relationship was found between the four subtypes of breast cáncer and PCR when the axillary node status was included in the analysis. A pCR rate of $21.1 \%$ $(15 / 71)$ was recorded. The phenotypes that produced the best response to treatment were TN subtypes $(40.0 \%$ of pCR) followed by luminal B and HER2 (16.7\%) and finally the luminal A subtype (12.5\%). There were not significant differences in pCR of tumor and axillary node status when histopathologic subtypes were grouped and compared to TN subtypes (40\% vs $16.1 \%$, p: 0071$)$.

Prognostic information obtained from the classification of breast cancer by histopathologic subtypes

During the follow up period of 45 months, the two deaths observed and four of the six recurrences corresponded to the TN phenotype. We compared the evolution of the different histologic phenotypes and observed that the TN tumours had a significantly lower rate of DFS $(p=0.022)$. At 30 months' follow up, the DFS rate was $71.8 \%$ in TN vs $93.3 \%$ in the other patients. These differences were also significant for OS $(p=0.006)$, with $100 \%$ of the non-NT patients alive vs. $85.7 \%$ of the TN patients, at 30 months' median follow up (Figures 1a,b). During the observation period of this study, the median level of OS or DFS was not reached, and so this parameter could not be calculated.

Prognostic value of $p C R$ in the different subtypes of breast cancer treated with dose-dense NCT

Cox's stratified analysis was used to identify the differences in OS and DFS in the different subtypes of breast cancer, according to whether pCR (Miller \& Payne classification grade 5) was achieved, compared to other pathologic responses (grades 1 to 4). Only the TN tumours presented a significant relationship between PCR and DFS, the latter being greater among patients with $p C R$ grade $5(p=0.048)$. OS and DFS at 30 months were both $100 \%$ in the TN patients who achieved pCR after NCT (Figures $2 a, b$ ). In the other subtypes, there were no differences in OS or DFS between patients who had achieved pCR and those who had not (data not shown). The median level of OS or DFS was not achieved in the non-NT patients.

It was not possible to perform a multivariate analysis, adjusting for other variables of known prognostic and predictive value, such as age or the $\mathrm{T}$ or $\mathrm{N}$ stages, because there were too few events for each study variable.

\section{Discussion}

Although diverse preoperative polychemotherapyschemes are used in routine practice, most include anthracyclines and taxanes administered sequentially every 21 days [14]. In order to optimise the effectiveness of NCT and thus increase the rate of $\mathrm{pCR}$, it has been proposed that chemotherapy should be applied every 14 days (in a densedose regimen). This is a proven treatment option, and is endorsed in clinical guidelines (for example, ESMO and NCCN). Nevertheless, it is not implemented as a standard treatment in all healthcare centres $[15,16]$.

In this prospective observational study, we analyse the effectiveness of NCT administered every 14 days during the first four cycles. We decided to administer only dose-dense administration for the first part (anthracycline-based), because of the risk of a significant increase in toxicity [16]. A pCR rate of $25.4 \%$ was recorded, a Figure comparable to those reported in studies evaluating NCT with three-weekly treatment regimens including anthracyclines and taxanes. One such study was the phase III NSABP30 B-27, with 2411 patients and 26\% pCR among those who received four cycles of anthracycline followed by four cycles of docetaxel [14]. Studies of NCT with DD anthracyclines and taxanes have reported lower rates of $\mathrm{PCR}$ than ours, while the superiority of the experimental arm, with higher rates of pCR, has been reported in others $[17,18]$. Nevertheless, our results should not be compared directly with others, as its study population was heterogeneous, with diverse NCT regimens in terms of number of cycles and type of cytotoxic agents, and because many previous studies did 


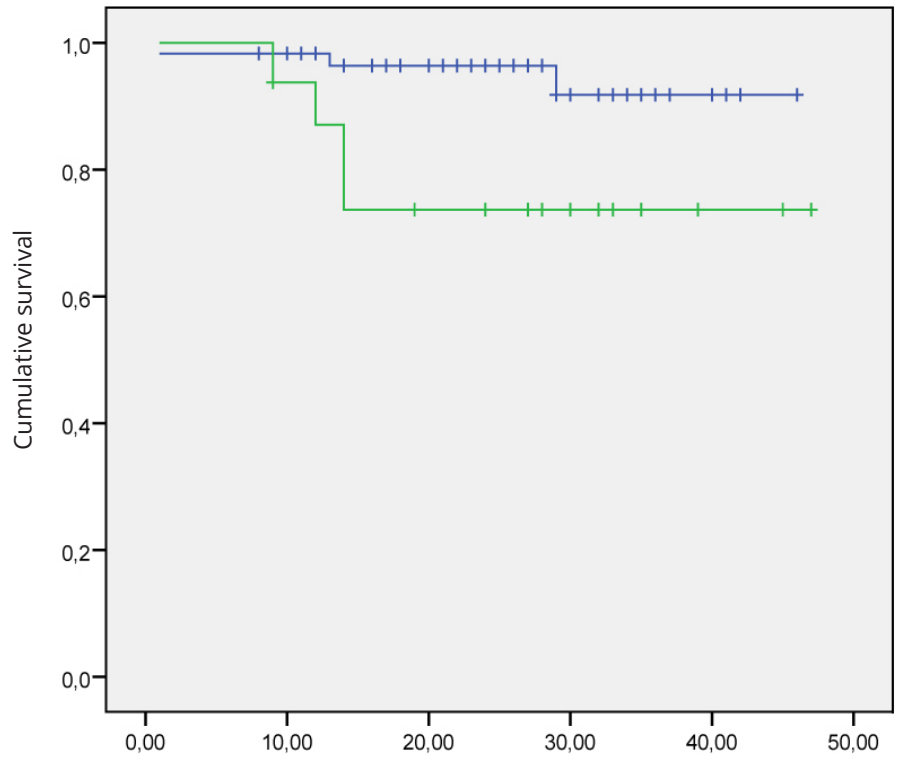

(a) Disease-free survival time

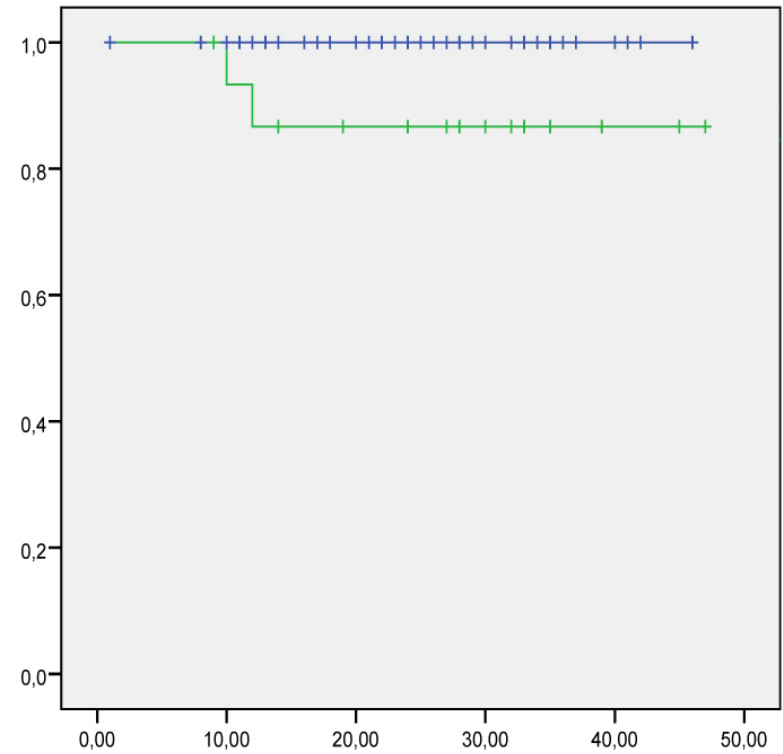

(b) Overall survival time

Figure 1a,b Kaplan Meier survival curves for breast cancer histopathologic subtypes. Green line: Triple negative population; Blue line: Others subtype; + Censored case.

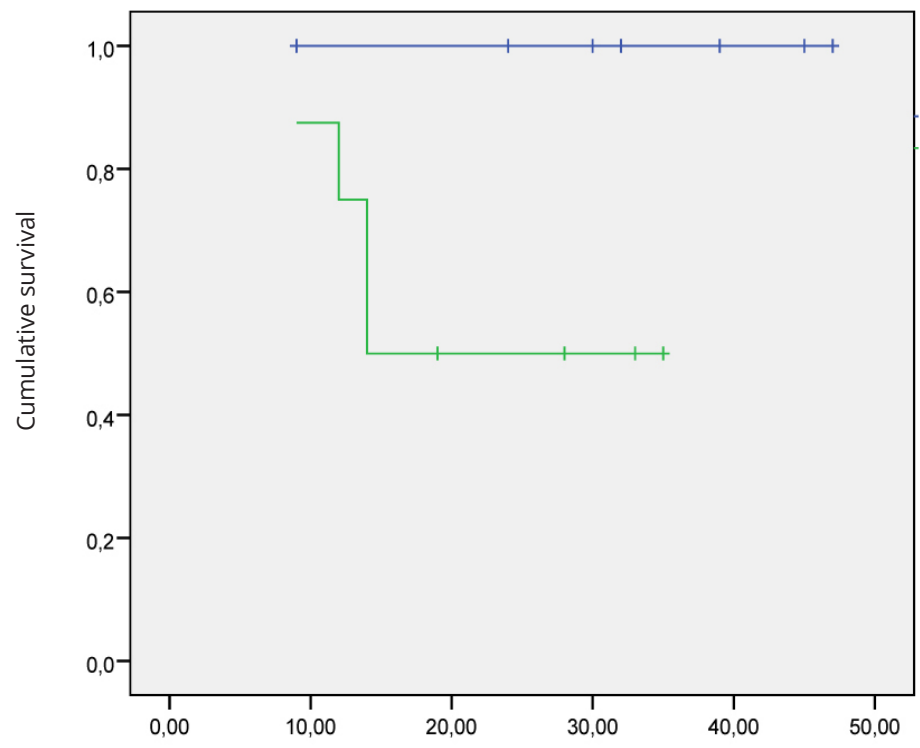

(a) Disease-free survival time

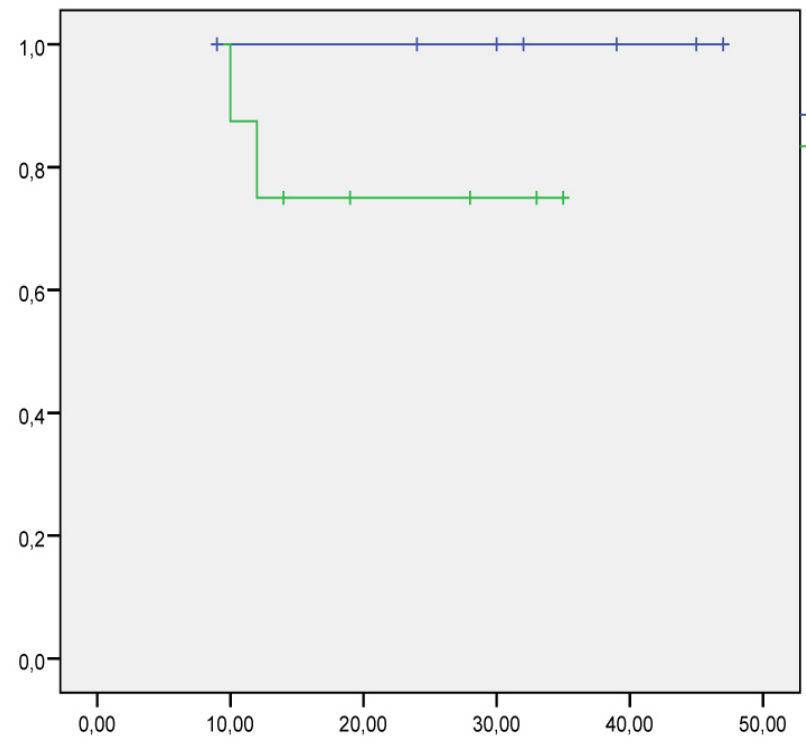

(b) Overall survival time

Figure 2a,b Kaplan Meier survival curves for triple negative subtype breast cancer according to Miller \& Payne pathologic response. Green line: Grade1-4 Miller \& Paine; Blue line: Grade5 Miller \& Paine (pCR); + Censored case.

not include neoadjuvant trastuzumab in patients with overexpressed HER2. However, we can conclude that our treatment regimen is effective, achieves a high rate of $\mathrm{pCR}$ and lies within the upper range of previously-published results.

In previous research, using DNA microarrays, breast cancer has been classified into molecular subtypes with different patterns of gene expression and varying levels of prognosis [19]. The predictive value of these subtypes for response to NTC has also been demonstrated, and a relation has been established between the TN and pure HER2 + molecular subtypes and a greater chance of achieving pCR, compared with luminal subtypes [20]. However, this predictive and prognostic relationship has not been clearly determined for DD NTC. Unfortunately, in daily practice this genotyping assessment is not readily available, and therefore our aim is to analyse the predictive value of the response to DD NTC and the prognostic value of the classification into four intrinsic subtypes according to the clinical and pathologic criteria set out in the St. Gallen consensus. St Gallen 2011 classification was employed, because our prospective study was designed in 2011 by applying the current histological classification at that time. And one of the strengths of our study is that the phenotype classification, by immunohistochemical analysis, was planned prospectively.

The results of our analysis are consistent with the above-cited molecular classification studies, revealing a significant relationship between the classification by the four immunophenotypes and the $\mathrm{PCR}$ for the tumour. 
The phenotypes that produced the best response to treatment were HER2 and TN, followed by luminal B and, finally, luminal $A$. The rate of $\mathrm{pCR}$ for the TN subgroup was noticeably higher than for the others, at $46.7 \%$ and $19.6 \%$, respectively. This higher rate of response in tumours with the TN immunohistochemical phenotype (HER2 negative and tumour with no expression of hormone receptors) was previously observed in the GeparTrio prospective trial [21], which obtained a pCR rate of $36.7 \%$ for the TN phenotype. Similarly, the GeparQuattro phase III clinical trial, with 445 HER2 positive tumours, using immunohistochemical analysis and FISH, obtained a pCR rate of $45.5 \%$, which was significantly higher than the $19.5 \%$ obtained for the HER2 negative phenotype [22]. Unfortunately, the number of patients with the pure HER2 phenotype in our study was insufficient for us to determine its predictive value for pCR.

Another limitation of this study was our decision not to include the axillary node status in the analysis of pathologic responses, despite the known prognostic role of observed residual disease in a pathologic examination of resected axillary lymph nodes $[8,9]$. However, this information was not available regarding patients enrolled during the first years of the study, and so it was decided to use only the Miller \& Payne assessment of the primary tumour. But in the following years, the authors aware of this limitation, decided to include the nodal status in this pathologic response evaluation, although no significant relationship was found between the four subtypes of breast cancer and pCR.

In our study, the median follow up period was 45 months, and evaluation showed the TN tumours to have significantly lower rates of DFS and OS than the other subtypes. Therefore, although TN tumours are the most sensitive to chemotherapy, producing the highest rates of pCR, they obtain lower rates of survival than the other, less aggressive, subtypes. We believe this is probably because the intrinsic biology of the tumour makes it more aggressive in patients who do not achieve PCR.

Doubt remains as to the prognostic value of $\mathrm{pCR}$ from breast cancer achieved after NCT. Although most studies conclude that pCR correlates with a better prognosis, and it has been considered a surrogate marker of OS in TN and pure HER2 molecular subtypes, various trials and meta-analyses have not succeeded in proving this association $[5,6]$. These discrepancies may be due to the methodological limitations of the trials, the heterogeneity of the population, the absence of a uniform definition of pCR (in some cases it is applied solely to the tumour and in others also to the axillary node), the use of different score systems (such as residual cancer burden, or the Miller \& Payne classification) and/or the existence of diverse NCT regimens (in some, trastuzumab is not employed in patients with overexpressed HER2). Therefore, in recent years many studies have searched for biomarkers of response to different NCT systems in this scenario [23].

In our study population, the TN subjects who obtained grade 5 pCR, according to the Miller \& Payne classification, after DD NCT achieved higher rates of DFS and OS than those with other types of response. However, these findings should be interpreted with caution because a more extensive follow up of the study population is needed in order to determine the extent of differences in the other subtypes for which there were insufficient events in the present study. Although this study has not a large number of patients, however, this sample was sufficient to achieve the objective of the study, therefore we found that immunohistochemical classification into four breast cancer subtypes has both prognostic and predictive value of response to dose-dense NCT. The possible beneficial impact on the prognosis for patients with TN who achieved pCR after DD NCT was also apparent in the three-weekly NCT treatment regimen. This shows that, within the poor prognosis for TN patients, those who achieve pCR after dose-dense NCT do live longer, and this association between $\mathrm{PCR}$ and improved survival was observed both for patients with TN tumours and for those with the HER2 immunophenotype, as defined by immunohistochemical criteria [8]. Furthermore, studies of the S-phase fraction have concluded that ER-negative tumours are associated with a low grade of differentiation and with greater proliferative activity of the cell cycle. Similarly, the presence of a high proportion of tumour cells in the S-phase of the cell cycle has been associated with response to treatment [24]. Therefore, in our search to optimise NCT regimens against aggressive tumours, the DD approach is an interesting option, given the hypothesis for this model of cell growth that there exists a higher proportion of replicating cells and therefore increased sensitivity to anthracyclines; thus, a reduced intercycle interval would limit the capabilities for cellular regrowth and increase cell death.

\section{Conclusion}

The immunohistochemical classification into intrinsic subtypes of breast cancer has both a predictive and a prognostic value for response to dose-dense NCT by TN phenotype patients who achieve PCR in the tumour after treatment.

\section{Conflicts of interest}

Authors declare no conflicts of interest.

\section{References}

[1] Fisher B, Gunduz N, Saffer EA. Influence of the interval between primary tumor removal and chemotherapy on kinetics and growth of metastases. Cancer Res. 1983; 43(4):1488-1492.

[2] Simpson-Herren L, Sanford AH, Holmquist JP. Effects of surgery on the cell kinetics of residual tumor. Cancer Treat Rep. 1976; 60(12):17491760.

[3] Mauri D, Pavlidis N, loannidis JP. Neoadjuvant versus adjuvant systemic treatment in breast cancer: a meta-analysis. J Natl Cancer Inst. 2005; 97(3):188-194.

[4] Fisher B, Bryant J, Wolmark N, Mamounas E, Brown A, et al. Effect of preoperative chemotherapy on the outcome of women with operable breast cancer. J Clin Oncol. 1998; 16(8):2672-2685.

[5] Van der Hage JA, Van de Velde CJ, Julien JP, Tubiana-Hulin M, Vandervelden C, et al. Preoperative chemotherapy in primary operable breast cancer: results from the European Organization for Research and Treatment of Cancer trial 10902. J Clin Oncol. 2001; 19:42244237.

[6] Rastogi P, Anderson SJ, Bear HD, Geyer CE, Kahlenberg MS, et al. Preoperative chemotherapy: Updates of National Surgical Adjuvant Breast and Bowel Project Protocols B-18 and B-27. J Clin Oncol. 2008; 26(5):778-785. 
[7] Insa A, Chirivella I, Llunch A. Neoadjuvant therapy for operable breastcancer. Med Clin (Barc). 2006; 126(8):295-303.

[8] Von Minckwitz G, Untch M, Blohmer JU, Costa SD, Eidtmann H, et al. Definition and impact of pathologic complete response on prognosis after neoadjuvant chemotherapy in various intrinsic breast cancer subtypes. J Clin Oncol. 2012; 30(15):1796-1804.

[9] Goldhirsch A, Winer EP, Coates AS, Gelber RD, Piccart-Gebhart M, et al. Personalizing the treatment of women with early breast cancer: highlights of the St Gallen international expert consensus on the primary therapy of early breast cancer. 2013. Ann Oncol. 2103; 24(9):2206-2223.

[10] Goldhirsch A, Wood WC, Coates AS, Gelber RD, Thürlimann B, et al. Strategies for subtypes--dealing with the diversity of breast cancer: highlights of the St.Gallen International Expert Consensus on the Primary Therapy of Early Breast Cancer 2011. Ann Oncol. 2011; 22(8):1736-1747.

[11] Hammond ME, Hayes DF, Dowsett M, Allred DC, Hagerty $K L$, et al. American Society of Clinical Oncology/College of American Pathologists guideline recommendations for immunohistochemical testing of estrogen and progesterone receptors in breast cancer. J Clin Oncol. 2010; 28(16):2784-2795.

[12] Wolff AC, Hammond ME, Schwartz JN, Hagerty KL, Allred DC, et al. American Society of Clinical Oncology/College of American Pathologists guideline recommendations for human epidermal growth factor receptor 2 testing in breast cancer. J Clin Oncol. 2007; 25(1):118-145.

[13] Ogston KN, Miller ID, Payne S, Hutcheon AW, Sarkar TK, et al. A new histological grading system to assess response of breast cancers to primary chemotherapy: prognostic significance and survival. Breast. 2003; 12(5):320-327.

[14] Bear HD, Anderson S, Brown A, Smith R, Mamounas EP, et al. The effect on tumor response of adding sequential preoperative docetaxel to preoperative doxorubicin and cyclophosphamide: preliminary results from National Surgical Adjuvant Breast and Bowel Project Protocol B-27. J Clin Oncol. 2003; 21(22):4165-4174.

[15] Bonilla L, Ben-Aharon I, Vidal L, Gafter-Gvili A, Leibovici L, et al. Dosedense chemotherapy in nonmetastatic breast cancer: a systematic review and meta-analysis of randomized controlled trials. J Natl Cancer Inst. 2010; 102(24):1845-1854.

[16] Lemos Duarte I, da Silveira Nogueira Lima JP, Passos Lima CS, Deeke Sasse A. Dose-dense chemotherapy versus conventional chemotherapy for early breast cancer: a systematic review with meta-analysis. Breast. 2012; 21(3):343-349.

[17] Untch M, Konecny G, Ditsch N, Sorokina Y, Moebus V, Muck B, et al. Dose-dense sequential epirubicin-paclitaxel as preoperative treatment of breast cancer: results of a randomised AGO study. Proc Am Soc Clin Oncol. 2002; 1:34.

[18] von Minckwitz G1, Raab G, Caputo A, Schütte M, Hilfrich J, Blohmer $\mathrm{JU}$, et al. Doxorubicin with cyclophosphamide followed by docetaxel every 21 days compared with doxorubicin and docetaxel every 14 days as preoperative treatment in operable breast cancer: the GEPARDUO study of the German Breast Group. J Clin Oncol. 2005; 23 (12):26762685.

[19] Perou CM, Sorlie T, Eisen MB, van de Rijn M, Jeffrey SS, et al. Molecular portraits of human breast tumours. Nature. 2000; 406(6797):747-752.

[20] Ayers M, Symmans WF, Stec J, Damokosh Al, Clark E, et al. Gene expression profiles predict complete pathologic response to neoadjuvant paclitaxel and fluorouracil, doxorubicin, and cyclophosphamide chemotherapy in breast cancer. J Clin Oncol. 2004; 22(12):2284-2293.

[21] Von Minckwitz G, Kümmel S, Vogel P, Hanusch C, Eidtmann H, et al. Neoadjuvant vinorelbine-capecitabine versus docetaxel-doxorubicincyclophosphamide in early nonresponsive breast cancer: phase III randomized GeparTrio trial. J Natl Cancer Inst. 2008; 100(8):542-551.

[22] Untch M, Rezai M, Loibl S, Fasching PA, Huober J, et al. Neoadjuvant treatment with trastuzumab in HER2-positive breast cancer: results from the GeparQuattro study. J Clin Oncol. 2010; 28(12):2024-2031.

[23] Wang J, Buchholz TA, Middleton LP, Allred DC, Tucker SL, et al. Assessment of histologic features and expression of biomarkers in predicting pathologic response to anthracycline-based neoadjuvant chemotherapy in patients with breast carcinoma. Cancer. 2002; 94(4):3107-3114.

[24] Remvikos Y, Mosseri V, Zajdela A, Fourquet A, Durand JC, et al. Prognostic value of the S-phase fraction of breast cancers treated by primary radiotherapy or neoadjuvant chemotherapy. Ann N Y Acad Sci. 1993; 698:193-203. 